\title{
Loa Representada en Ibagué para la Jura del Rey Fernando VI ${ }^{1}$
}

$E^{L}$ original de la loa y del poema considerados en este estudio, compuestos por Jacinto de Buenaventura, forma parte de la colección y biblioteca del doctor Guillermo Hernández de Alba, de Bogotá, Colombia. Consta la loa de cinco páginas de texto y a ella sigue el corto poema descriptivo de 84 versos en romance en las páginas 7 y 9, dejando en blanco las páginas 6 y 8 . Faltan partes de algunos versos en las páginas $1,2,3$ y 4 , resultado de la pérdida de las esquinas de dos hojas. Revela dicho poema que la loa fué compuesta para inaugurar con una laudatoria la función miscelánea de la jura del Rey Fernando VI, comenzada en Ibagué (hoy capital del Departamento del Tolima) el día 8 de septiembre ${ }^{2}$ de $1752^{3} \mathrm{y}$ prosiguiendo "siete días con muchos toros / y un día de fiesta yntermedio" (Poema, versos 63-64). E1 Alférez Real Fernando Joseph Caicedo ${ }^{4}$ aprontó lo necesario para la fiesta.

Después de la misa salió del Convento de Santo Domingo la figura de la Virgen con su estandarte en la mano, previniendo a todos la jura para esa misma tarde, para la que constituyeron el acompañamiento cuatro Reyes. En la plaza habian levantado un teatro de tres gradas embellecidas de finas telas "a las que tapaba el techo/ de una media naranxa, / la que estauan guarneciendo / quatro hermosos gallardetes / y por remate un supremo" (Poema, versos 48-52). Colgaduras decoraban las casas recién blanqueadas de la alegre población. Por la noche hubo una pandorga ${ }^{5}$ con luminarias y un entremés. Los días restantes de la fiesta fueron celebrados con toros, el fingido ataque a un castillo, con lucha entre moros y españoles, saraos, pandorgas, entremeses, comedias y otras diversiones. ${ }^{6}$ 
El título de la loa, aunque incompleto, nos da a conocer el nombre del poeta Jacinto de Buenaventura y descubre a la vez que fué compuesta para honrar, con motivo de la jura, al Rey y al Alférez Real. Hay seis personas que hablan, además de la Música y de los cuatro moros que, como criados, sirven para conducir en hombros al Rey a la escena. Las acotaciones especifican que éstos dejen al Monarca "deuajo de su citial" (Loa, acotaciones). Luego se le rinde homenaje cantando unos versos de adoración y de invitación a venir a verle. El Embajador de las cuatro partes del mundo (Europa, Africa, Asia y Mérica) ${ }^{7}$ informa al Rey que éstas desean dedicarle a sus enemigos, los cuatro moros. E1 Rey les concede audiencia, y a poco vuelve a aparecer el Embajador, esta vez acompañado de los moros amarrados y de las cuatro damas, con armas en las manos. Hablan por turno las señoras, cada uná ofreciendo su vencido prisionero al Rey Fernando VI, el que movido por amor y piedad perdona a sus antiguos enemigos, a quienes manda desatar. Los moros se arrodillan y después bailan y cantan de alegría. Se disputan las damas el privilegio de ensalzar al bondadoso Monarca. El Embajador aconseja que se otorgue este honor a Mérica, quien habla con fervor de los bienes que resultaron al mundo por su coronación. El primero de los moros se había atrevido a negarle la Prudencia, el segundo la Justicia, el tercero la Fortaleza y el cuarto la Templanza. Prueba su defensora que el Rey posee las cuatro virtudes cardinales, cualidades atribuidas al caballero perfecto desde la Edad Media. ${ }^{8}$ En la conclusión de las palabras de Mérica el poeta Buenaventura se refiere a sí mismo diciendo que como leal sujeto procura obsequiar al Soberano. En esto se disparan las armas y se tocan las chirimías. ${ }^{2}$ Después las cuatro damas congratulan a Ibagué por tener entre sus vecinos al noble Alférez Real Fernando Joseph Caicedo, de Bogotá, quien con este lucido acto de jura trae gloria al pueblo y honor al Rey. Rinde el autor tributo a la belleza de la esposa del señor Caicedo. $\mathrm{Y}$ termina la loa con las gracias que expresa Fernando VI a la Ciudad. Otra vez se tocan las chirimías y se disparan las armas y se corre la cortina de la escena.

$\mathrm{La}$ loa, de sencillo lenguaje y de poco artificio, ofrece escaso valor literario, pero, no obstante, tiene interés para la historia del histrionismo y de las costumbres en Colombia, donde son algo escasos los datos del teatro de ese período. Esta composición literario-musical y el poema nos ayudan a formar una impresión de la vida local y de 
las diversiones de esa sociedad, desaparecida ha casi dos siglos, cuando solemnizaron un acontecimiento histórico, la jura de un Monarca y el festejo de un Alférez Real, con representaciones teatrales, hechas más atractivas con bailes y ornamentos musicales. Estos festejos también manifiestan cómo a mediados del siglo xviIr ciertas prácticas de España habían penetrado hasta los pueblos más aislados de las colonias. En cierto sentido la loa y el poema pueden considerarse verdaderos cuadros de costumbres.

La loa está compuesta de 311 versos, octosílabos todos con excepción de uno (verso 5) y divididos en 224 versos llanos y 87 agudos. Unas dos terceras partes de los versos están en quintillas (algunas tienen rimas defectuosas), estrofa usada con más frecuencia en las loas de los autos primitivos. ${ }^{10}$ Hay muchos cantos en la pieza, versos en gran parte formados de dos estribillos que alternan con los recitados por las damas que simbolizan cuatro partes del mundo. Escasea el aparato escénico, limitado a un sitial para el Rey, unas andas para llevarle a la éscena, la cortina, las armas de las damas y las chirimías.

El poeta, en su afán patriótico, procura glorificar en frases convencionales al imperio español como defensor de la fe católica, sin admitir que España hubiera dejado de ser la poderosa nación de antaño ni reconocer la flaqueza de los Borbones. Con su loa intenta tejer - sin preocuparse por convertirla en fábula dramática- una corona poética al Soberano acudiendo al usual recurso de introducir personajes alegóricos, práctica general ya degenerada en amaneramiento, que cantan la gloria real pero sin decir nada concreto con respecto a su carácter, figura o vida, costumbre igualmente común en obras de esta clase. ${ }^{11} \mathrm{~A}$ lo menos en una ocasión Buenaventura consigue expresarse en un "concepto discreto", reminiscente de los poetas gongorinos, en el cual descubre de manera ingeniosa el nombre de la esposa del Alférez, doña Teresa Flores, y el de su pueblo natal, Vélez: "traiendo un xardín de flores / con lo florido de Beles" ( $L o a$, versos 269-270). Muéstrase el autor bastante diestro en introducir las virtudes cardinales negadas al Rey por los moros, y en su alusión a la joya perdida (Loa, versos 138-147) se vale de términos que recuerdan las parábolas narradas por Jesucristo (Lucam, 15). Y asimismo en el poema que describe los festejos se refiere a varios sucesos de la Biblia: el maná, los cinco panes con que Cristo dió de 
comer a la multitud y los víveres despachados a David y sus cuatrocientos soldados.

Pág. 1. Música que Dió al Rey Nuestro Señor y al Alferes Real [Fernando] de Caizedo Don Jasinto de Buenaventura en la [Jura]

Personas que hablan:

E1 Rei

Embajador

Asia

Europa

Africa

Mérica

Quatro moros y éstos llebatán al Rei en ombros disiendo y cantando. Dejan el Rei detajo de su citial y las quatro partes del mundo y los moros se entran dentro con el Embajador.

$1^{\circ}$

$2^{\circ}$

$3 \circ$

$4^{\circ}$

Todos.

Música.

Todos.

Embajador.

¡Contento, goso, plazer!

¡Alegría, gusto, consuelo!

¡Complasencia, regosijo!

iCon nuestro Rei mucho es esto!

iVengan a verlo, vengan a verlo!

Las quatro partes del mundo

con alegría duplicada

dizen en hunidas boses:

¡viua nuestro Rei de España!

¡Viua nuestro Rei de España!

Sacro y superio[r] Monarca,

a vuestra presencia vengo,

como Embaxador nombrado

de quatro hunidos afectos:

Europa, Africa, Asia

y la Mérica, queriendo

dedicar a vuestra [s] plantas

quatro enemigos opuestos

que a vuestra noble persona

violauan vuestro respecto.

Rei. Buelue, Embajador, y di

que aquí aguardo su festejo.

Embaxador. Benigno Señor, estimo

vuestra lisencia y corriendo

boi a desirles que bengan

a obsequiaros luego, luego.

Música. ¿Viua, viua para siempre!

Vaze.

¡Ay, qué goso y qué contento,

tiene el orue por tener

por Rei un Fernando Sexto!

30

Todos.

¡Por Rei un Fernando Sexto! 
Sale el Emuajador $y$ saca a los moros amatrados y salen las cuatto damas con armas en las manos.

Embajador. Has de rendir la servís

a nuestro Rei respectando

en ausencia y en presencia

su onor y respecto sacro.

Europa. Yo soi la Europa, Señor.

..... cio mi afecto y valor

uno de aquellos traidores

ollando sus pundonores

[y] lo traigo prisionero para que os sirua el primero

y se aumenten tus onores.

Música.

¿Viua, viua para siempre!

iAy, qué goso y qué contento

tiene el orbe por tener

por Rei un Fernando Sexto!

Todos. $\quad$ Por Rei un Fernando Sexto!

Asia.

Escucha, Monarca ymbicto:

yo soi vuestra amante la Asia,

que con amor y eficazia

por vuestro onor solizito

y conosiendo el delito

de uno de éstos en negarte

la obediencia al ynstante,

le bensí y le aprizioné

$y$ os lo traigo pata que

humilde os sirua constante.

60

Música. ¿Viua, viua para siempre!

¡Ay, qué goso y qué contento

tiene el orbe por tener

por Rei un Fernando Sexto!

Todos.

¡Por Rei un Fernando Sexto:

Africa.

Oie, potestad exselsa:

yo soi la Africa y postrada

a tu presencia eleuada

ofresco a vuestra grandeza

una victoriosa presa,

que estando a bos resistente

mi cuidado dilixente

le sujetó y le venció,

la que os traigo alegre yo

a tus plantas a ofreserte. 
Música. ¡Viua, viua para siempre!

¡Ay, qué goso y qué contento

tiene el orbe por tener

por Rei un Fernando Sexto!

Todos. ¿Por Rei un Fernando Sexto!

Mérica. Señor, la Mérica soi,

que rendida a tu obediencia

sujetó mi dilijencia

un atrevido que os doi

a que te sirua desde oy

vuestros órdenes guardando

$y$ viua reberenciando

a tu Alteza soberana

$y$ que su osadía ufana

os sirua y esté adorando.

Música. ¡Viua, viua pata siempre!

¡Ay, qué goso y qué contento, tiene el orbe por tener, por Rei un Fernando Sexto!

Todos. ¡Por Rei un Fernando Sexto!

Rei. Vuestra obediencia agradesco

y perdono su osadía.

Desataldos y que vean

el amor y piedad mía.

Los desata el Embajador disiendo:

Embajador. Perdón hauéis meresido,

el fabor reconosed,

besad a su Magestad

sus sacros piadosos pies.

Pág. 3. Los quatro se ponen de rodillas y dizen y luego bailan disiendo:

Los quatro. El perdón agradesemos

y a vuestra planta postrados

desimos con todo gusto:

iviua nuestro Rei Fernando!

Europa. $\quad$ A mi es a quien pertenese

a nuestro Rei elojiar.

Asia. Yo soi la de ese lugar;

Africa. Vuestra resolusión sese,

que yo soi la que e de ser.

y así e de mereser

de aquesta suerte dichosa. 
Mérica. A una Deidad magestuosa

a mí perteneze hablar

$y$ a sus pies sacrificar

mi voluntad afectuosa.

Embajador. La Europa el derecho seda, la Asia ventaja no pida, y que se dé por vensida la Africa para que pueda la Mérica alegre y queda en deleitosa quietud ensalsar a la virtud de nuestro grande Monarca por set de prendas una arca con tan rejia esadtitud.

Las 3. Alegres obedesemos

a vuestra gran direcsión

y con gustoza atención

tu desempeño lo oiremos.

Música.

¡Viua, viua para siempre!

¿Áy, qué goso y qué contento

tiene el orbe por tener

por Rei un Fernando Sexto!

Mérica. ¿Qué gran [gozo y qué placer]

quando una joia per[dida]

buelue a ser conseguid[a]

de quien la dejó perder,

y se deja conoser

por hauerse ya perdi[do]

y por eso a conseguido

más apresio de su dueño,

por lo qual con más empeño

a más apresio a subido!

Lo mismo a susedido, supremo y grande Fernando, pues, estando suspirando

con el dolor estendido, todo el orbe a conseguido con vuestra coronazión gusto, plazer, galardón, amparo, sombra, efujio con tan rejia protecsión. Por lo que con atención $y$ con afecto postradas oy benimos animadas a rendirle adorazión, 
Pág. 4.

y muestra de aquésta son aquellos quatro despojos, que les quebramos los ojos defendiendo a tu deidad, pues a vuestra puridad no se han de atreber arrojos.

Vno de ellos se atrevía, opuesto a vuestra prudencia y con belós ymprudencia, de aquesta virtud desía

él, que no la conosía, vuestra sacra dignidad siendo esto de calidad que desde tu tierna ynfancia prudencia con elegancia

[muestra] Vuestra Magestad.

[La] virtud de la justizia desía el segundo no hallaua en bos y esto blasonaba con desembuelta malizia.

Salgan aquí la milizia, litigantes $\mathrm{y}$ acredores y brillarán resplandores de aquesta virtud en bos, pues da por tu mano Dios castigos, premios, onores.

El terzero arrojado desía no hauía fortaleza en vuestra sacra nobleza quando se a esperimentado el que hanéis sujetado al tedio, a la osadía con tan bisarra ydalguía, floresiendo esta virtud en tu ynfancia, en jubentud y en ahumento cada día. E1 quarto atrevido moro con furiosa destemplanza desía que la templanza no se hallaua en tu tesoro, siendo así que a tu decoro esta virtud te a adornado, siendo en todo tan templado, tan compuesto y tan medido que de tu exemplo a aprendido 
el modesto más mirado.

Hasta aquí pudo llegar

la obligación de mi empleo,

supliendo mi gran deseo

lo que no puedo alcanzar.

Dios, Señor, te haga reinar

años muchos y dichosos

$y$ que en delizias y gosos

sea una continua victoria

hasta llegar a la gloria,

trono de los benturosos,

y siendo Buenaventura, quien festejaros procura, como leal a tu corona dedica a tu real perzona

con liberalidad pura

este cortejo, deseando

el orbe te esté obsequiando

y que diga en cada asaña:

Iviba nuestro Rei de España,

Todos. IViba nuestro Rei de España,

viba nuestro Rei Fernando!

Dispatan y tocan chitimias.

Música. iVictor, ylustre Ciudad;

victor, mui noble Cabildo;

víctor, Ybagué, que alegre

a Fernando dises víctor!

Europa. Oie, Ciudad de Ybagué,

que te doi los parauienes

de que en tu senado tienes

quien te ame con tanta fe,

como al presente se ve,

vuestros onores buscando

con lusimientos jurando

a nuestro Rei con empeño,

siendo en esto el desempeño

un caballero Fernando.

Música. iVíctor, ylustre Ciudad;

víctor, mui noble Cabildo:

victor, Ybagué, que alegre

Pág. 5. Asia. Plásemes mui repetidos

os doi, Ybagué famoza, 
por veros tan obstentosa

con adornos tan lusidos,

todos éstos conseguidos

por la mano liberal

de un noble Alferes Real

que medios a bido poniendo,

mas bástale ser Caizedo

Música.

para que sea tan cabal.

iVictor, ylustre Ciudad;

víctor, mui noble Cabildo;

víctor, Ybagué, qué alegre

Africa. Con vuestro gusto e formado

O, Ybagué, grande contento, pues con tanto lusimiento el recreo has abumentado con beros tan adornado

con tan Iusidos laureles,

cauzando esto un grande Apeles

con matises y colores

traiendo un xardín de flores

con lo flotido de Beles.

Música. iVíctor, ylustre Ciudad;

víctor, mui noble Cabildo;

víctor, Ybagué, que alegre

a Fernando dises víctor!

Mérica. Dichoso Ybagué, gosad

de vuestro gusto y plazer,

no seses de floreser,

antes en prosperidad

vuestra delizia ahumentad,

pues tenéis de Santa Fe

quien tantos lauros te dé,

y que sea pública bos

que si dichas te ymbió Dios

son por Fernando Joseph.

Música. iVictor, ylustre Ciadad; víctor, mui noble Cabildo; víctor, Ybagué, que alegre

a Fernando dizes víctor?

Embajador. ¡Viua esta ylustre Ciudad, brille este noble Cabildo

de Ybagué, porque gustoso

a Fernando dise víctor! 
Todos. IViba esta ylustre Ciudad, brille este noble Cabildo de Ybagué porque gustoso

Rei. A Fernando dize víctor! Estimo hunidos afectos los aplausos que hauéis dado a quien por Rei me a jurado benerando mis respectos con tan lusidos efectos los que no se han de oluidar sino siempre han de brillar porque uean que $\mathrm{mi}$ corona saue premiar la persona que me sabe culto dar.

Europa. Que se cumpla así, Señor.

Asia. Lo apresiamos y queremos,

Africa. $\quad Y$ en su casa dejaremos

Mérica. Ympresas con todo amor

Todas.

Disparan y tocan chitimías y al Rei le corten la cortina.

Pág. 6. En blanco.

Pág. 7.

Descripción de los festejos

No ay que desconfiar de Dios

en desabrigos therrenos,

que sabe dar de sus vienes

y a manos llenas lo vemos,

pues liberal manifiesta

su piedad en todos tiempos

como se vió en el maná.

jeneral mantenimiento.

Así se vió en sinco panes

que sólo ellos mantubieron

a tanto número de hombres

que hambre estauan padeziendo.

Asi mantubo a Dabid

con soldados quatrosientos.

$Y$ así lo a manifestado

en tantos casos diuerzos,

y así to a esperimentado

Ybagué, pues padesiendo

grande desdicha y pobreza,

y presisado al empeño 
de obsequiar a nuestro Rei,

Dios guarde, Fernando Sexto, estando en este conflicto ymbió Dios al desempeño a un noble Alferes Real, Don Fernando de Caisedo, quien con su modo galante prebino con grande acuerdo la jura a nuestro Monarca, arbitrando y disponiendo.

el día 8 de septiembre

la fiesta en el Combento del Padte Santo Domingo, después de misa saliendo la General Capitana y Emperatrís de los cielos con su estandarte en la mano listando y preainiendo a todos para la tarde ber jurar al Rei therreno: lo que se bizo tan sumptuoso con alarde vien compuesto, quatro Reies mui bisarros, lusido acompañamiento, vn grande teatro en la plaza de tres gradas que vistieron las más escojidas telas. a las que tapaba el techo de una media naranxa, la que estauan guarneciendo quatro hermosos gallardetes $y$ por remate un supremo. Las puertas y las bentanas con colgaduras de presio, todas las casas blanqueadas, las calles con mucho aseo y los muchachos alegres coxiendo plata del suelo. A la noche una pandorga con luminarias y fuegos y un presioso entremés. Deste modo prosiguieron siete días con muchos toros y un dia de fiesta yntermedio un abanze de un castillo con los moros y los nuestros, 
no faltando entremeses,

saraos, entretenimientos,

pandorgas, buenas comedias

$y$ otros lusidos festejos

70

Pág. 8. En blanco.

Pág. 9. que ymbentaron los vesinos

para maior lusimiento.

Bendito sea Dios por siempre

que cría jenerosos pechos

para honrar a nuestro Rei

75

alentando, adbirtiendo

para que todos quedazen

lusidos y mui contentos.

Rei del cielo, dadle vida

a tu Fernando, pues de ello

las ciudades lograrán

un benefactor tan bueno,

y después de muchos vienes

dalde la gloria por premio.

\section{NOTAS}

\section{INTRODUCCION}

1 Para el American Council of Learned Societies de Washington, D. C., que me otorgó una beca para realizar mis estudios en Colombia durante el verano de 1942, así como para el doctor Guillermo Hernández de Alba, que tuvo la bondad de poner a mi disposición su ejemplar de la loa y de proporcionarme unas notas relativas a la fecha de la ceremonia y a la esposa del Alférez Real, mi gratitud más cordial, bien merecida.

2 Véase el poema que describe los festejos, verso 31.

3 Fecha suministrada por mi amigo el profesor Guillermo Hernández de Alba.

4 Fernando José de Caicedo y Vélez Ladrón de Guevara, natural de Santa Fe de Bogotá, como revela la loa (verso 280), fué hijo de don José de Caicedo y Pastrana y de doña Mariana Vélez Ladrón de Guevara. Su hermana doña María Clemencia fundó el primer colegio de niñas que existió en Nueva Granada. Su hermano Manuel de Caicedo Vélez Ladrón de Guevara sirvió los destinos de rector y catedrático en teología en el Colegio del Rosario y en 1748 se hizo comisario del Santo Oficio por el tribunal de Cartagena. Nuestro Alférez Real contrajo matrimonio con doña Teresa Flores y Olarte, nacida en Vélez (capital del Departamento de Santander), como dice la loa (versos 267-270). Uno de sus hijos fué Fernando de Caicedo y Flores, es- 
critor y primer arzobispo de la república. Una de las familias más distinguidas y mejor consideradas de Colombia fué la de los Caicedo, hallada en las Indias desde el primer tercio del siglo XVI. Véase Alberto y Árturo García Carraffa, Enciclopedia hetáldica y genealógica hispanoamericana, Madrid, 1925, XXI, 2526: Ignacio Gutiérrez Ponce, Crónicas de mi hogat en la época colonial (1536 a 1816), London and Tonbridge, 1926, págs. 538 y 560; Pedro M. Ibáñez, Ctónicas de Bogotá, 2a edición, Bogotá, 1913, I, 369-374; Arturo Quijano, "E1 arzobispo prócer", Boletín de historia y antigüiedades, Bogotá, 1932, XIX, 410-411; José María Vergara y Vergara, Historia de la literatuta en Nueva Granada desde la conquista hasta la independencia (1538-1820), 3a edición, Bogotá, 1931, I, 384-387; Joaquín Ospina, Diccionatio biogtáfico y bibliográfico de Colombia, Bogotá, 1927, I, 381-382, 384; Guillermo Hernández de Alba, Crónica del muy ilustre Colegio Mayor de Nuestra Señora del Rosario en Santa Fe de Bogotá, Bogotá, 1940, II, 45-46, 127-136, 169-172, 257.

5 ". . las pandorgas constituían un número de los festejos populares que se organizaba con alguna especie de orden; y así, por ejemplo, con motivo de las fiestas y regocijos con que se celebró el nacimiento de Felipe IV en Valladolid, el Ayuntamiento acordó 'que para que la máscara se haga con grande aparato de ruido y regocijo, vaya en ella una Fuga de danzas de diferentes maneras y una Pandorga por la orden y traza que diere el señor don Luis de Alcaraz a quien esta ciudad nombra...' (14 de abril de 1605)." Véase Enciclopedia universal ilustrada, Barcelona, XLI, 751.

6 Para descripciones de las juras de Luis I (1724), de Fernando VI (1747), de Carlos IV (1790) y de Fernando VII (1808), unidas a las fiestas pomposas con los alborotos del pueblo, riegos de grandes cantidades dé dinero, fuegos artificiales, iluminaciones, suntuosos bailes y banquetes, músicas, toros, paseos a caballo de la nobleza, vistosas cuadrillas, misas solemnes, representaciones "de comedias, loas, etc., véase Gutiérrez Ponce, op. cit., 579-582; Ibáñez, op. cit., I, 272-274, 290; "La jura de Carlos IV en Cali"; "La jura de Carlos IV en Timaná"; Raimundo Rivas, "La jura de Fernando VII (en Bogotá)":; "Relación de la augusta proclamación del señor don Fernando VII . . ejecutada en esta villa de San Bartolomé de Honda el 25 de diciembre de 1808"; José Vicente París Lozano, "La jura de Fernando VII (en Ibagué, en 1816)"; Boletín de historia y antigüedades, Bogotá, 1909, v, 159-167, 725-737; 1913, VIII, 117-120, 637-645; 1916, XI, 655-657.

7 En varias loas (Loa para la primer flor de el Carmelo, Loa para llamados y escogidos, Loa para los encantos de la culpa), así como en estos dos autos escritos por Calderón de la Barca ( $E l$ valle de la zarzuela, La semilla y la cizaña) salen personificadas las cuatro partes del mundo. Véase Pedro Calderón de la Barca, Autos sactamentales alegóricos y histotiales, ed. por Juan Fernández de Apontes, Madrid, 1759-1760, 6 tomos. Agustín de Salazar y Torres hace comparecer las cuatro partes del mundo en su loa palaciega para la comedia Elegit al enemigo; esta loa fué compuesta para celebrar cantando y con una danza el cumplimiento de los tres años de Carlos II, en 1664. Las cuatro partes del mundo figuran también en una loa que fué representada con la comedia El alcázat del secteto en el cumpleaños del rey don Pedro II, hacia 
1687, en Lisboa; está en español y sirve para cantar loores a los soberanos. Véase Colección de entremeses, loas, bailes, jácatas y mojigangas desde fines del siglo XVI a mediados del XVIII, ordenada por Emilio Cotarelo y Mori, NBAE, Madrid, 1911, XVII, xxxvi, xl.

8 Ramón Lull (1235-1315?), en su explicación del significado del equipo del caballero señala entre otras cualidades las virtudes cardinales. Véase Le libre del orde de cauayletia, traducida al inglés por William Caxton, de una versión francesa (Early English Text Society), London, 1925, CLXVIII, 76-89. Alfonso el Sabio afirma que el rey, lo mismo que el caballero, debe estan dotado de dichas cuatro virtudes. Véase Las siete partidas, Madrid, 1807, Part. II, 'Tít. V, Ley VIII, Tít. XXI, Ley IV, vol. II, 30-31, 200. Las cuatro partes de la espada simbolizan para él estas propiedades: "Et porque los defensores non habríen todos comunalmente estas armas, et aunque las hobiesen non podríen siempre traerlas, tovieron por bien los antiguos de facer una en que se mostrasen todas estas cosas por semejanza, et ésta fué la espada. Ca bien así como las armas que el home viste para defenderse muestran cordura, que es virtud quel guarda de todos los males que le podríen avenir por su culpa, otrosí muestra eso mismo el mango de la espada que el home tiene encerrado en su puño; ca en quanto así lo toviere, en su poder es de alzarla, o de baxarla, o de ferir con ella o de la dexar. Et otrosí como en las armas que el defendedor para ante sí para defenderse muestra fortaleza, que es virtud que face a home estar firme a los peligros que le avienen, así en la manzana es toda la fortaleza de la espada, ca en ella se sufre el mango et el arriaz et el fierro. Et bien como las armaduras que el home ciñe son medianeras entre las armas que viste et las armas con que fiere, et son así como la virtud de la mesura entre las cosas que se facen además o de menos de lo que deben, bien a esa semejanza es puesto el arriaz entre el mango et el fierro della. Et bien otrosí como las armas que el home tiene en las manos endereszadas para ferir con ellas allí do conviene muestran justicia que ha en sí derecho et egualdat, otrosí lo muestra el fierro de la espada que es derecho et agudo et taja egualmente de amas partes. Et por todas estas razones establescieron los antiguos que la troxiesen siempre consigo los nobles defensores et que con ella rescebiesen honra de caballería, et non con otra arma, porque siempre les veniese emiente destas quatro virtudes que deben haber en sí; ca sin ellas non podríen complidamente mantener el pueblo del defendimiento para que son puestos." Ibid., Ley IV, págs. 200-201. Antoine de la Sale (1388 - fallecido después de 1469), en su Petit Jehan de Saintré, IX, trata de la educación del paje aspirante a ser caballero e indica que a él le hacen falta las virtudes cardinales y teologales. Véase Raoul Rosières, Histoire de la société française au moyen-age (987-1483), 3ème edition, Paris, 1884, I, 381 382. Alonso Jerónimo de Salas Barbadillo también inviste a su caballero perfecto de las cuatro virtudes cardinales. Véase El caballero perfecto, Madrid, 1620. Baldessar Castiglione, además de rendir alto tributo a las citadas virtudes, afirma que su caballero perfecto debe implantarlas en el alma de los príncipes. Véase Il libro del cortegiano, Milano, 1928, págs. 362 y 372.

9 Chirimía - "Instrumento músico de madera encañonado a modo de trompeta, derecho, sin vuelta alguna, largo de tres quartas, con diez agujeros 
para el uso de los dedos con los quales se forma la harmonía del sonido según sale el aire. En el extremo por donde se le introduce el aire con la boca tiene una lengüeta de caña llamada pipa, para formar el sonido y en la parte opuesta una boca mui ancha como de trompeta, por donde se despide el aire." Véase Diccionario de Autoridades.

10 En el siglo XVII el romance llegó a ser el metro favorito para la loa. De las 34 loas en verso de Agustín de Rojas Villandrando, 23 están en romance. Véase Lope de Vega Carpio, Obras, publicadas por la Real Academia Española, Madrid, 1892, II, xxv y passim; Joseph A. Meredith, Introito and Loa in the Spanish Drama of the Sixteenth Century, Philadelphia, 1928, págs. $104-$ 108; Harry Warren Hilborn, A Chronology of the Plays of D. Pedto Calderón de la Barca. Toronto, 1938, págs. 79-113, passim.

11 Véase Colección de entremeses, loas, etc., xxxviii.

\title{
LOA'
}

Versos 138-147. Lucam, cap. 15.

Verso 163. Les sacaron los ojos a Sansón y a Sedecías. Véase Judicum, $16: 21$ y Jetemias, $39: 7,52: 11$.

Versos 167-206. Para comentarios sobre las virtudes cardinales véase Plato, The Republic. Bk. IV y Laws, Bk. I (Dialogues), translated into English by B. Jowett, 3a edición, Oxford, 1892, III, 117-139; IV, 7; Santo Tomás, Summa Theol., I-II, Q. Ixi, aa. 2 y 4; Augustinus, De Moribus Ecclesiae Catholicae, XV.

Verso 267. Apeles fué el más ilustre de los pintores griegos (siglo IV antes de Jesucristo).

\section{POEMA}

Versos 7-8. Exodus, cap. 16.

Versos 9-12. Mattheum, 14:17-21.

Versos 13-14. I Regum, cap. 25.

Verso 23. Se repite este verso en la margen izquierda del manuscrito.

Versos 35-36. La figura de la Virgen María.

Verso 79 , dadle aquí pero dalde en verso 84 , ejemplo de metátesis.

\author{
HARVEY L. JoHnSON, \\ Northwestern University, \\ Evanston, Illinois.
}

